\title{
UCP1 expression-associated gene signatures of human epicardial adipose tissue
}

\author{
Kanta Chechi, ${ }^{1,2}$ Jinchu Vijay, ${ }^{3}$ Pierre Voisine, ${ }^{2,4}$ Patrick Mathieu, ${ }^{2,5}$ Yohan Bossé, ${ }^{2,6}$ Andre Tchernof, ${ }^{2,7}$ \\ Elin Grundberg, ${ }^{8}$ and Denis Richard ${ }^{1,2}$ \\ 'Department of Medicine, Faculty of Medicine, Université Laval, Québec, Québec, Canada. ²Institut universitaire de \\ cardiologie et de pneumologie de Québec (IUCPQ), Chemin Sainte-Foy, Québec, Canada. ${ }^{3}$ Department of Human Genetics, \\ McGill University, and Genome Québec Innovation Centre, Montreal, Québec, Canada. ${ }^{4}$ Department of Cardiovascular \\ Surgery, ${ }^{5}$ Laboratory of Cardiovascular Pathobiology, Department of Surgery, Faculty of Medicine, and ${ }^{6}$ Department of \\ Molecular Medicine, Université Laval, Québec, Québec, Canada. 'School of Nutrition, Université Laval, Québec, Québec, \\ Canada. ${ }^{8}$ Children's Mercy Kansas City, Kansas City, Missouri, USA.
}

Multiple reports of uncoupling protein 1 (UCP1) expression have established its presence in human epicardial adipose tissue (eAT). Its functional relevance to eAT, however, remains largely unknown. In a recent study, we reported that adrenergic stimulation of eAT was associated with downregulation of secreted proteins involved in oxidative stress-related and immune-related pathways. Here, we explored the UCP1-associated features of human eAT using next-generation deep sequencing. Paired biopsies of eAT, mediastinal adipose tissue (mAT), and subcutaneous adipose tissue (sAT) obtained from cardiac surgery patients, with specific criteria of high and low expression of UCP1 in eAT, were subjected to RNA sequencing. Although eAT exhibited a depot-specific upregulation in the immune-related pathways relative to mAT and sAT, high UCP1 expression in EAT was specifically associated with differential gene expression that functionally corresponded with downregulation in the production of reactive oxygen species and immune responses, including $T$ cell homeostasis. Our data indicate that UCP1 and adaptive immunity share a reciprocal relationship at the whole-transcriptome level, thereby supporting a plausible role for UCP1 in maintaining tissue homeostasis in human eAT.

Conflict of interest: The authors have declared that no conflict of interest exists.

Copyright: () 2019 American Society for Clinical Investigation

Submitted: July 16, 2018 Accepted: March 7, 2019 Published: April 18, 2019

Reference information: /CI Insight. 2019;4(8):e123618. https://doi. org/10.1172/jici.insight.123618

\section{Introduction}

Enhanced accumulation of fat around the heart is a well-established risk factor for the development of cardiovascular disease (CVD), coronary artery disease (CAD), atrial fibrillation, and heart failure $(1,2)$. Accumulation of fat in the cardiac region occurs both inside and outside the pericardium in fat depots labeled as the epicardial and the paracardial, in essence, mediastinal adipose tissue (mAT), respectively. However, the unique anatomic location of epicardial adipose tissue (eAT) where no fascia-like structure separates the epicardial adipocytes from the myocardium, its reported invaginations into the myocardium (3), as well as its shared blood supply with the myocardium clearly place eAT in a unique position to influence cardiac physiology in a vasocrine and paracrine manner (4). Indeed, a causal role for eAT in the development of $\mathrm{CAD}$, and hence its therapeutic targeting to manage CAD and CVD, has been proposed repeatedly $(2,5,6)$.

Human eAT expresses uncoupling protein $1(U C P 1)(7,8)$, a brown adipocyte-specific mitochondrial protein, which executes thermogenesis via uncoupling of ATP synthesis from fat oxidation during cold stress (9). Considering that thermogenic activity translates into enhanced energy expenditure and metabolic benefits $(10,11)$, there is a lot of interest in characterizing the physiological relevance of UCP1 in human fat depots. Indeed, both classic brown and beige fat depots have been identified in humans (12-15). In a recent study, we also observed variable yet consistent expression of UCP1 in the eAT of 53 cardiac surgery patients (15). In addition, eAT exhibited beige features and a functional thermogenic ability that shared a negative association with oxidative stress-related markers in our study (15). The objective of the current study was to extend our understanding of the UCP1-related features of human eAT in participants exhibiting either high or low eAT-specific UCP1 expression whose samples were selected from our previous cohort 
and subjected to next-generation RNA sequencing. Our study design included pairwise analyses of mAT and subcutaneous adipose tissue (sAT) derived from each of the selected patients, thereby allowing us to identify depot-specific transcriptomic signatures. In addition, we aimed to characterize the transcriptomic signatures of eAT, mAT, and sAT as a function of obesity or CAD.

Consistent with our previous observations, eAT exhibited a gene expression profile that was closer to mAT than sAT. Both eAT and mAT had gene expression profiles matching with an overrepresentation of immune-related pathways relative to sAT. Although obesity was associated with detectable transcriptomic shift in sAT alone, UCP1-related transcriptomic shift was unique to eAT. Interestingly, high expression of $U C P 1$ was associated with downregulation of adaptive immunity as well as oxidative stress-related genes. These observations support the concept that UCP1 plays a role in the maintenance of tissue homeostasis via the regulation of the immune system.

\section{Results}

\section{Clinical characteristics of the patients}

The primary criterion for sample selection was differential expression of UCP1 in eAT of patients undergoing heart surgery as previously reported (15). Specifically, we selected patients at the high $(n=5)$ and low $(n=5)$ end of the UCP1 expression spectrum based on quantitative PCR (qPCR) profiling for patients from whom a sufficient amount of high-quality RNA could be obtained. Next, we segregated patients into obese versus lean and $\mathrm{CAD}$ versus non-CAD (NCAD) groups. Clinical and anthropometric features of the 10 patients included in the study are given in Supplemental Table 1, A and B (supplemental material available online with this article; https://doi.org/10.1172/jci.insight.123618DS1). These characteristics are also provided when patients were segregated based on BMI, presence of CAD, and UCP1 expression (Supplemental Table 1, C-E).

\section{Depot-specific transcriptomic profiles of eAT, mAT, and SAT}

As eAT, mAT, and sAT samples were collected in all patients $(n=10)$, we first sought to identify depot-specific gene signatures, pathways, or networks in a pairwise manner. Using threshold criteria of fold change (FC) greater than or equal to 2 or less than or equal to -2 and FDR less than or equal to $0.05,1,367$ differentially expressed genes (DEGs) (936 upregulated and 431 downregulated) were identified in eAT relative to sAT (i.e., eAT vs. sAT group) (Figure 1A), 423 (394 upregulated and 29 downregulated) in mAT relative to sAT (i.e., mAT vs. sAT group) (Figure 1B), and 515 (210 upregulated and 305 downregulated) in eAT relative to mAT (i.e., eAT vs. mAT group) (Figure $1 \mathrm{C}$ ). Indeed, these data reveal that at the whole-transcriptome level, eAT is closer to mAT than sAT whereas mAT lies somewhere in the middle of eAT and sAT with relative proximity to sAT.

Among the top DEGs, eAT exhibited marked upregulation in the expression of GATA4, TCF21, and $T B X 20$, transcription factors associated with cardiac development and function, relative to both $\mathrm{mAT}$ and sAT (Figure 1, D and F), whereas eAT and mAT together exhibited an upregulation in the expression of genes involved in immune responses like PKHD1L1, CCL21, and STAB2 in addition to GATA4 relative to sAT (Figure 1, D and E). In contrast, sAT exhibited specific upregulation in the developmental genes $H O X B 8$ and $H O X A 10$ relative to both eAT and $\mathrm{mAT}$, whereas $\mathrm{mAT}$ and sAT together exhibited an upregulation in the expression of $E N 1$ and $I R X 1$ along with the homeobox (HOX) family of genes (i.e., HOXA5, HOXC6, HOXC8, and HOXC9), relative to eAT (Figure 1, D-F). Clearly, mAT and sAT exhibited much similarity in terms of top DEGs relative to eAT (Figure 1, D-F). Detailed descriptions of these genes and associated Gene Ontology (GO) pathways are given in Supplemental Table 2.

A similar pattern of divergence of eAT from sAT was apparent upon canonical pathway analysis (CPA) using Ingenuity Pathway Analysis (IPA) (QIAGEN Inc.; www.qiagenbioinformatics.com/products/ingenuity-pathway-analysis/), where a number of immune-related pathways, predominantly related to $\mathrm{T}$ cell responses, such as iCOS-iCOSL signaling in T helper cells $(P<0.0001)$, Th2 pathway $(P<0.0001)$, T helper cell differentiation $(P<0.001)$, and calcium-induced T lymphocyte apoptosis $(P<0.001)$, were enriched in eAT over sAT (Figure 2A). Commonality of eAT and mAT relative to sAT was obvious in the upregulation of additional immune-related pathways, such as T cell receptor signaling $(P<0.0001)$, CTL4 signaling in cytotoxic T lymphocytes $(P<0.0001)$, Th1 and Th2 activation pathway $(P<0.0001)$, and B cell development $(P<0.001)$ (Figure 2A). In addition, $\mathrm{mAT}$ exhibited an upregulation in pathways related to development (i.e., factors promoting 
cardiogenesis in vertebrates, $P<0.01$ ) and eNOS signaling (i.e., cellular effects of sildenafil, $P<0.001$ ) relative to sAT, whereas eAT exhibited an upregulation in GPCR signaling $(P<0.05)$ and eicosanoid signaling $(P<0.001)$ besides additional immune response-related pathways, such as leukocyte extravasation and signaling $(P<0.05)$ and complement system activation $(P<0.01)$, when compared with mAT (Figure 2A). sAT was predominantly characterized by an upregulation in xenobiotic metabolism-related pathways, such as acetone degradation I $(P$ $<0.0001)$, bupropion degradation $(P<0.001)$, melatonin degradation $\mathrm{I}(P<0.01)$, and nicotine degradation II $\&$ III $(P<0.001)$, whereas $\mathrm{mAT}$ had an upregulation in the cardiac $\beta$-adrenergic signaling pathway $(P<0.01)$, in eNOS signaling (i.e., cellular effects of sildenafil, $P<0.001$ ) and in adherence junction signaling (i.e., tight junction signaling, $P<0.001$; and actin cytoskeleton signaling, $P<0.01$ ) when compared with eAT (Figure $2 \mathrm{~B}$ ). A similar pattern of overrepresentation of immune-related processes appeared in eAT and $\mathrm{mAT}$ relative to sAT when the diseases and functions analysis tool of IPA was used to identify the altered functions in eAT versus mAT, eAT versus sAT, and mAT versus sAT groups (Supplemental Figure 1).

To further characterize the individual adipose tissue signatures/pathways, we performed Venn diagram analysis on all the detectable genes for eAT, mAT, and sAT (inclusion criterion: reads per kilobase of transcript per million mapped reads $[\mathrm{RPKM}] \geq 10$ for at least 1 patient for each adipose tissue). A total of $13,118,13,042$, and 12,835 genes were identified for eAT, mAT, and sAT, respectively, of which 284, 191, and 156 genes were unique to eAT, mAT, and sAT, respectively (Supplemental Figure 2A). Next, we looked at the top 25 genes with highest expression uniquely observed in each of these fat depots. Detailed descriptions of these genes and associated GO pathways are given in Supplemental Table 3. ITLN1 (RPKM $=17,477.8)$, IGKV3-20 (RPKM = 514.2), and SIM1 (RPKM = 548.8) were the top expressed genes in eAT, $\mathrm{mAT}$, and sAT, respectively. Expression of the top 2 to 3 uniquely expressed genes in each eAT, mAT, and sAT was further verified using a qPCR approach (Supplemental Figure 3, A-H), where most exhibited a consistent expression pattern. Specific upregulation in the expression of omentin (i.e., ITLN1) in eAT has been reported previously $(16,17)$. Clearly, most of the highly expressed genes in eAT and mAT belong to immune- and metabolism-related GO pathways, whereas development-related genes predominate in sAT (Supplemental Table 3). When categorized as functional protein classes, eAT and mAT exhibited an upregulation in kinases, transmembrane receptors, and GPCRs relative to sAT, whereas sAT exhibited an upregulation in transcriptional regulators among its uniquely expressed genes relative to eAT and mAT (Supplemental Figure 2B). In addition, enzymes and cytokines were overrepresented in eAT relative to $\mathrm{mAT}$ and sAT, whereas overrepresentation of miRNAs was characteristic of mAT compared with eAT and sAT (Supplemental Figure 2B).

IPA's CPA tool further identified 25, 13, and 7 pathways represented by genes uniquely expressed by eAT, mAT, and sAT, respectively (Supplemental Figure 2C). Indeed, an overrepresentation of T cell-related immune pathways, such as cytotoxic T lymphocyte mediated apoptosis of target cells $(P<0.01)$, calcium-induced $\mathrm{T}$ lymphocyte apoptosis $(P<0.01)$, CTLA4 signaling in cytotoxic T lymphocytes $(P<0.05)$, and Nur 77 signaling in T lymphocytes $(P<0.05)$, was specific to eAT relative to mAT and sAT. In addition, multiple lipid metabolism-related pathways commonly associated with thermogenesis, such as fatty acid activation $(P<0.01)$, mitochondrial L-carnitine shuttle pathway $(P<0.05)$, fatty acid $\beta$-oxidation I $(P<$ $0.05)$ and $\gamma$-linolenate biosynthesis $(P<0.01)$, were identified in eAT but not in mAT or sAT. In contrast, cell cycle-related pathways (i.e., cell cycle regulation by BTG family proteins, $P<0.05$; role of CHK protein in cell cycle check point control, $P<0.05$; and cell cycle G1/S checkpoint regulation, $P<0.05$ ) were identified in $\mathrm{mAT}$, whereas protein metabolism-related pathways (i.e., urea cycle, $P<0.05$; arginine degradation, $P$ $<0.05$; and citrulline biosynthesis, $P<0.05$ ) were uniquely represented in sAT (Supplemental Figure 2C). Venn diagram analysis further showed that 300,128 , and 145 targets were shared among eAT and mAT, eAT and sAT, as well as mAT and sAT, respectively (Supplemental Figure 2A). Clearly, eAT and mAT had more genes in common than eAT and sAT, indicating the proximity of 2 visceral fat depots over the subcutaneous fat depot. However, as a visceral fat depot, mAT was closer to sAT than eAT (Supplemental Figure 2A).

Obesity-associated major transcriptomic shift occurred in $s A T$. When segregated by BMI, we ended up with $n=5$ lean $(\mathrm{BMI} \leq 25)$ and $n=5$ obese (BMI $\geq 30)$ patients. This was associated with significant differences in BMI, body weight, and body surface area in our lean versus obese subgroups (Supplemental Table 1C). However, no differences were observed in other biochemical parameters, such as cholesterol and triglyceride levels, in these groups (Supplemental Table 1C).

Using threshold criteria of FC greater than or equal to 2 or less than or equal to -2 and FDR less than or equal to 0.05 , revealed 5,0 , and 46 DEGs (20 upregulated and 26 downregulated) whereas 

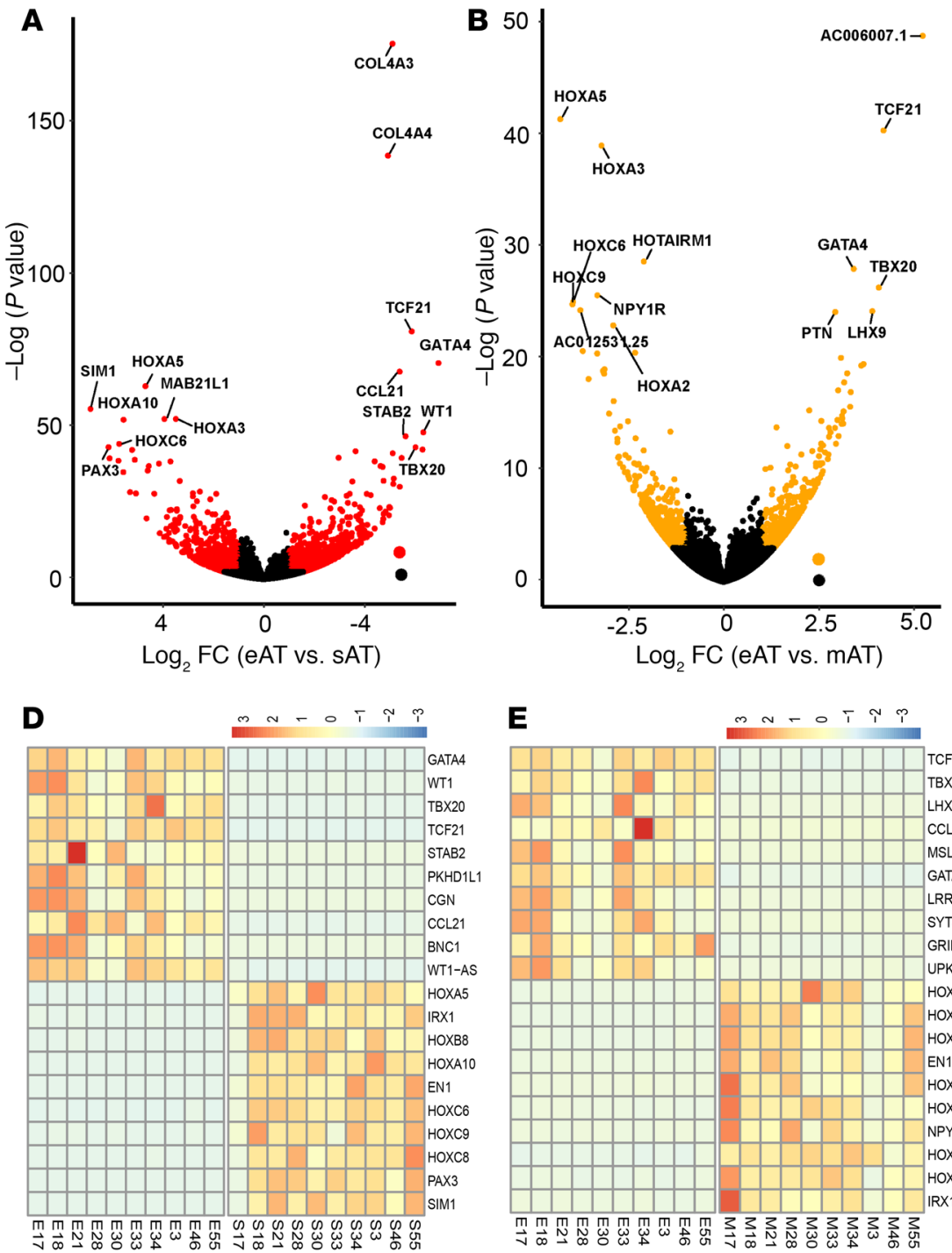

eAT vs. SAT

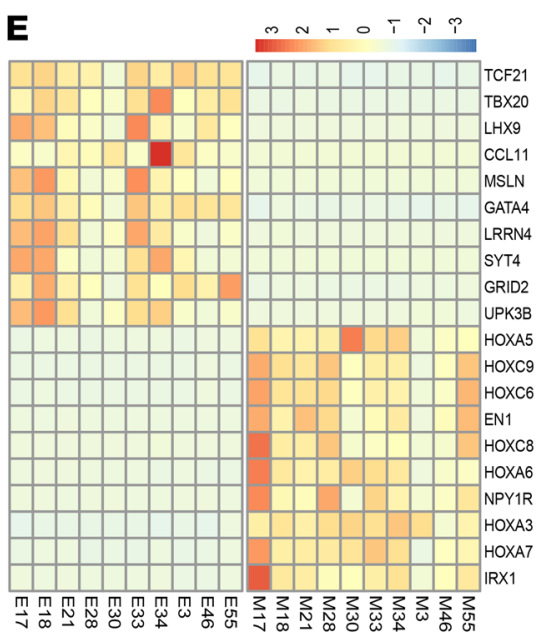

eAT vs. mAT

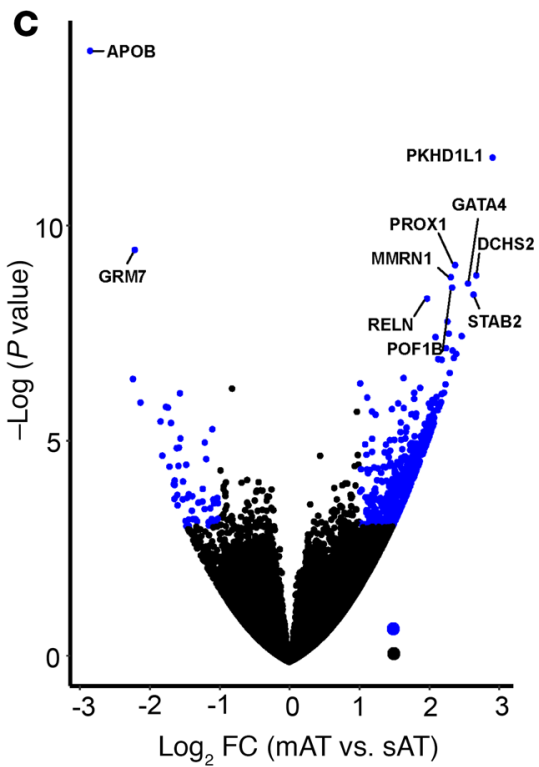

$\mathbf{F}$

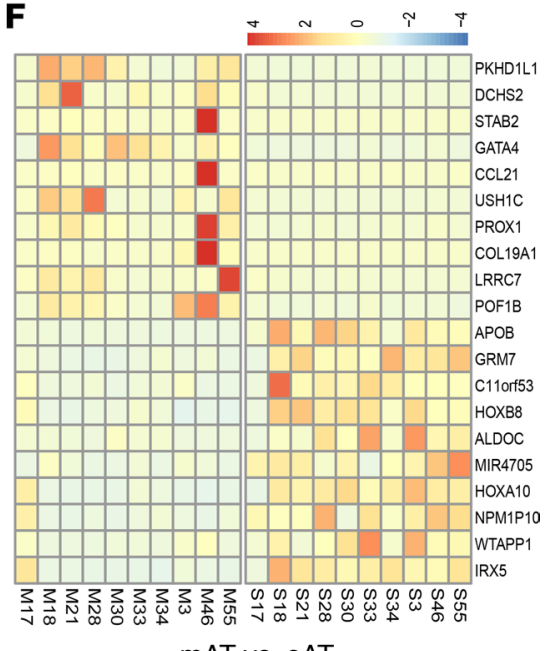

mAT vs. sAT

Figure 1. Depot-specific transcriptomic differences in eAT, $\mathbf{m A T}$, and sAT. Volcano plots revealing the DEGs (FC $\geq 2$ or $\leq-2 ; \mathrm{FDR} \leq 0.05)$ in (A) eAT versus sAT, (B) mAT versus sAT, and (C) eAT versus mAT groups. Heatmaps representing the individual expression (data scaled to row) of top 10 up and downregulated genes in (D) eAT versus sAT, (E) mAT versus sAT, and (F) eAT versus mAT groups.

using a threshold criterion of FDR less than or equal to 0.05 revealed 6, 1, and 108 DEGs in obese versus lean eAT, mAT, and sAT, respectively (Figure 3, A-C). Clearly, sAT exhibited most of the obesity-associated transcriptomic shift with an upregulation in 58 and downregulation in 50 genes under obese versus lean conditions, with top upregulation in genes involved in cell substrate adhesion processes and cytoskeleton organization, such as EGFL6, DDR1, and TUBB2A, and top downregulation in factors such as $B M P 3$ as a regulator of mesenchymal stem cell proliferation and adipogenesis (ref. 18 and Supplemental Figure 4C). Detailed descriptions of these DEGs and associated GO biological processes are included in Supplemental Table 4. To grasp the functional relevance of the transcriptomic shift in SAT, the upregulated and downregulated genes were subjected to Metascape analysis separately. GO terms such as regulation of cell substrate adhesion $(P<0.01)$ and response to growth factor $(P$ $<0.01)$ and the Reactome pathway HSP90 chaperone cycle for steroid hormone receptors $(P<0.0001)$ were observed in obese sAT whereas positive regulation of sodium ion transport $(P<0.0001)$ was the most significant GO term associated with lean sAT in our study (Supplemental Figure 4D). In contrast, when all the DEGs in sAT (i.e., 108) were subjected to IPA's CPA tool, pathways such as epithelial adherence signaling $(P<0.01)$, gap junction signaling $(P<0.05)$, complement system $(P<0.05)$, 
A

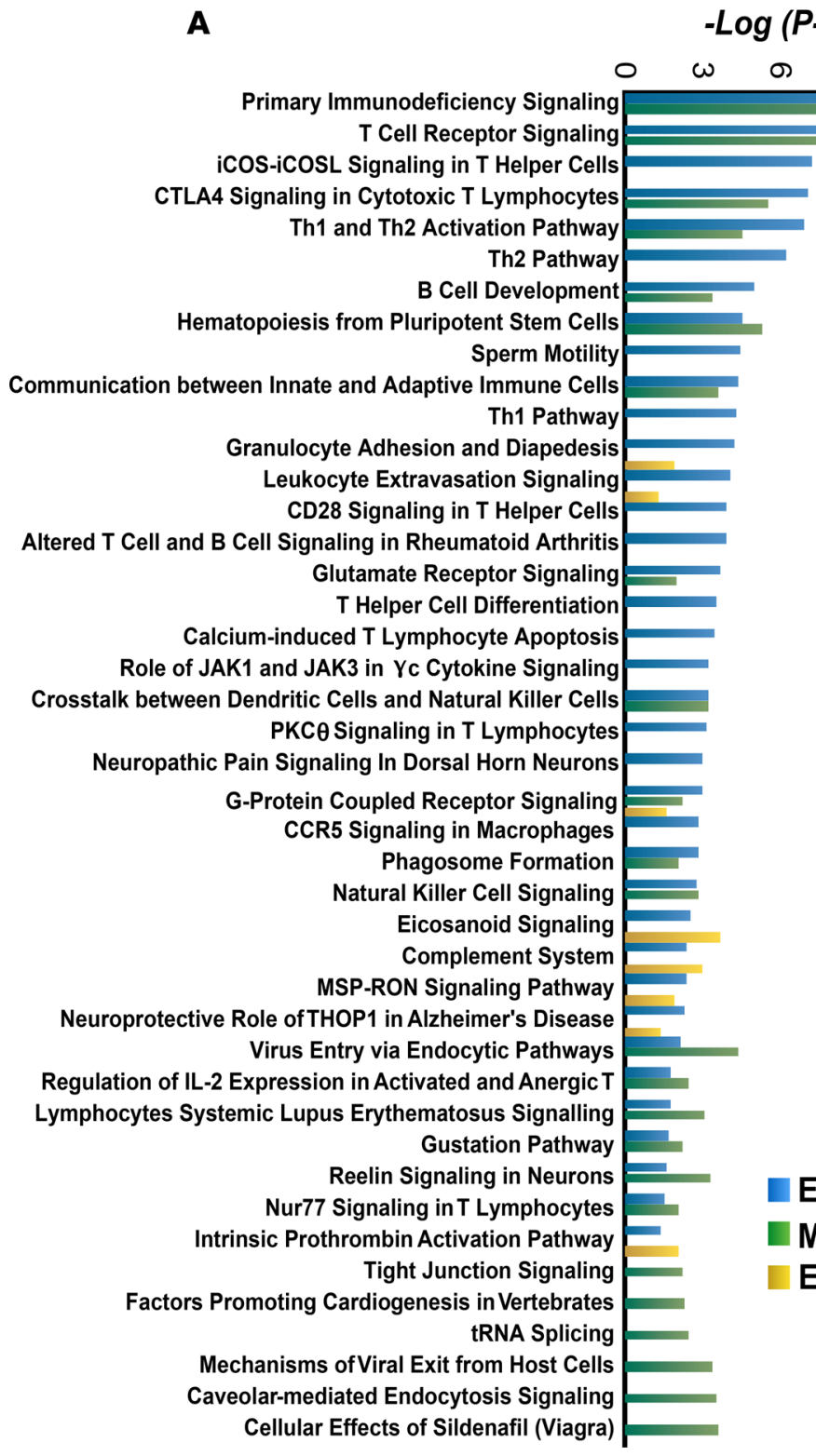

B -Log (P-value) $\vec{N}$

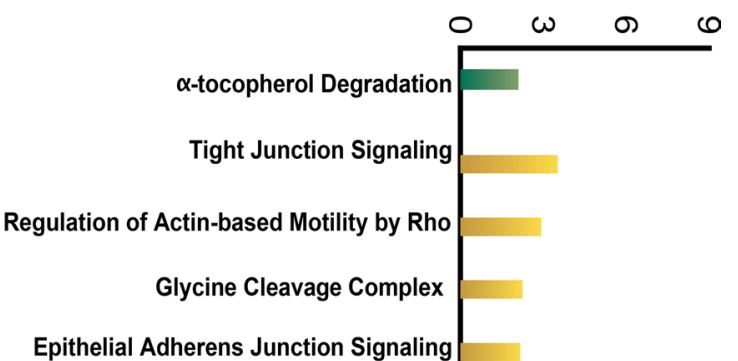

vs. S

M vs. S

E vS. M Factors Promoting Cardiogenesis in Vertebrates

Intrinsic Prothrombin Activation Pathway

Cellular Effects of Sildenafil (Viagra)

Figure 2. Proposed functional differences in eAT, mAT, and sAT. Top 25 canonical pathways associated with (A) up- and (B) downregulated genes in eAT versus sAT, mAT versus SAT, and eAT versus mAT groups exhibiting Benjamini-Hochberg-corrected $P$ value less than or equal to 0.05 identified using IPA's CPA tool.

PXR/RXR activation $(P<0.01)$, glycerol-3-phosphate shuttle $(P<0.05)$, and glutathione redox reactions II $(P<0.05)$ were represented in sAT (Supplemental Figure 4E).

Obesity was associated with selective downregulation in CEMIP, IGLV3-9, and NUGGC, which are genes associated with inflammatory responses, and upregulation in ADAM32 and LINC01347 in eAT (Supplemental Figure 4A and Supplemental Table 4), whereas SAMD9L, a gene involved in growth and maturation of cells, was the only upregulated gene identified in obese mAT (Figure 3E, Supplemental Figure 4B, and Supplemental Table 4).

$C A D$-associated major transcriptomic shift occurred in $m A T$. When segregated based on the presence $(n=6)$ or absence of CAD $(n=4)$, no differences were observed in any of the anthropometric or metabolic features of the patients (Supplemental Table 1D). Using threshold criteria of FC greater than or equal to 2 or less than or equal to -2 and FDR less than or equal to 0.05 revealed 1,27 , and 5 DEGs, whereas using a threshold criterion of FDR less than or equal to 0.05 identified 1,30 , and 7 DEGs in the CAD versus NCAD eAT (Figure 3D), mAT (Figure 3E), and sAT (Figure 3F), respectively. $\mathrm{CAD}$ had the most impact on $\mathrm{mAT}$, with 25 upregulated and 5 downregulated genes under $\mathrm{CAD}$ 
A

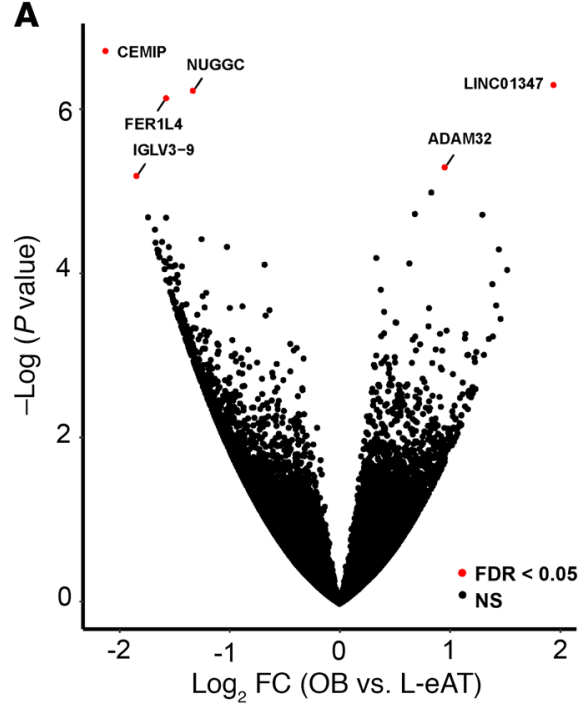

D

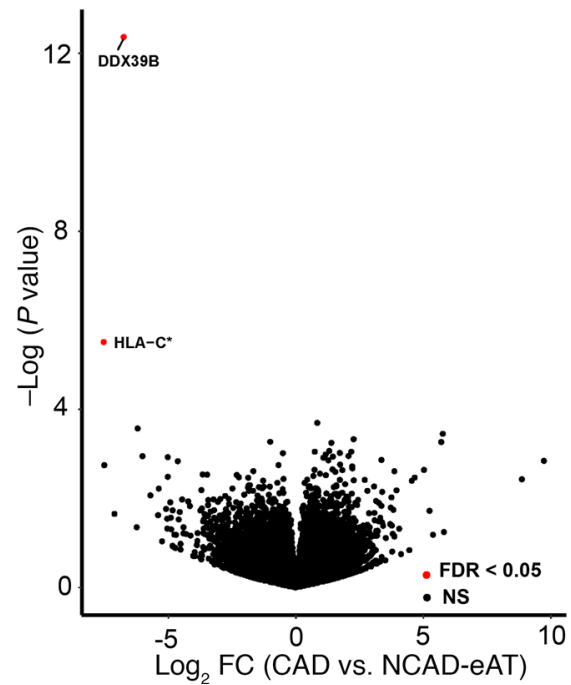

B

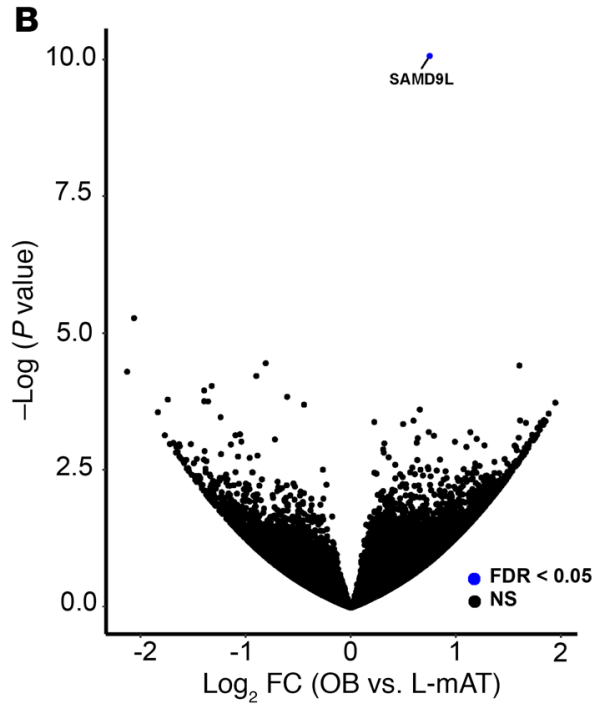

E

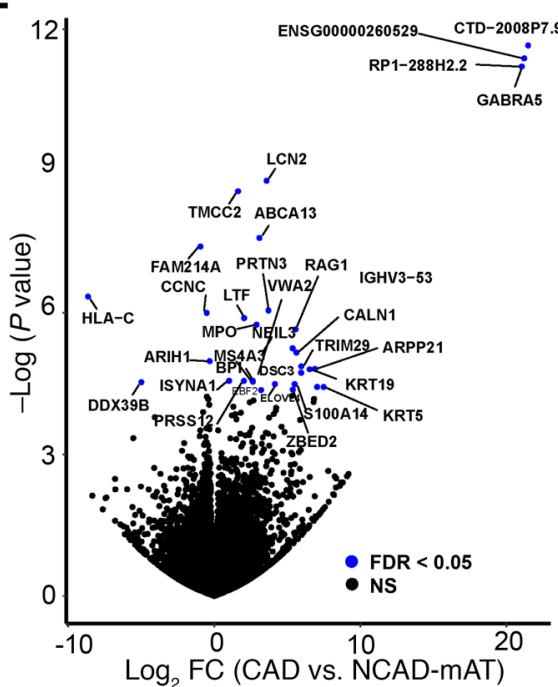

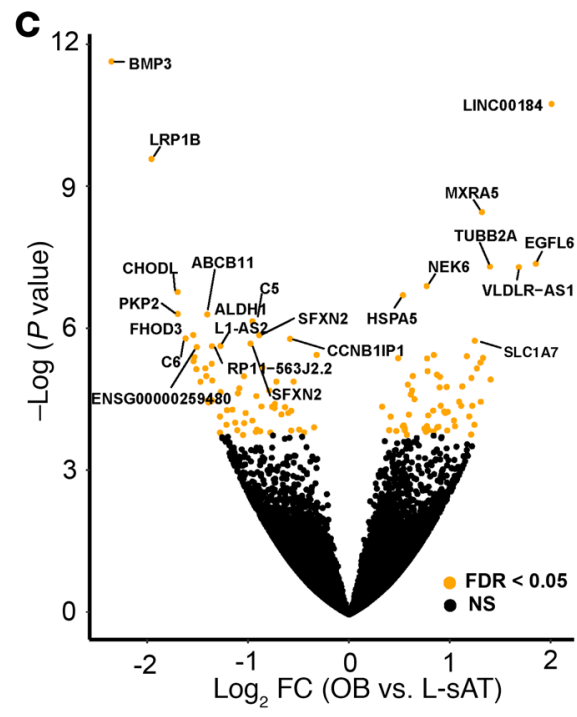

$\mathbf{F}$

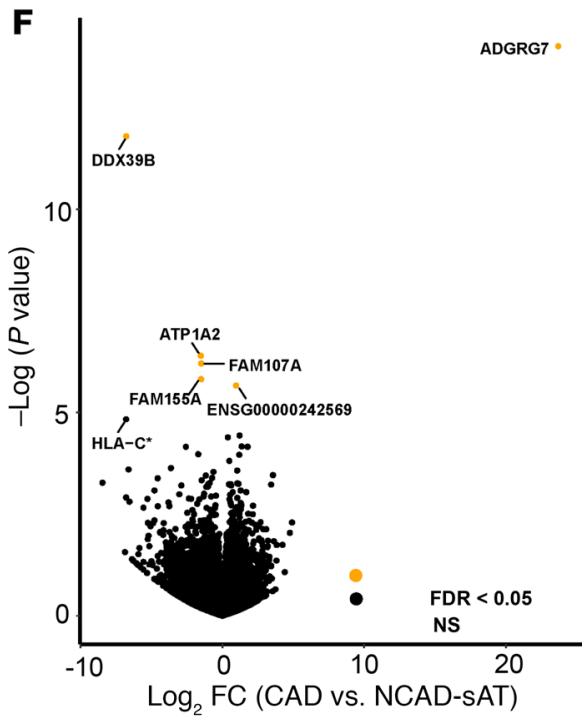

Figure 3. Obesity- and CAD-associated transcriptomic differences in eAT, $\mathbf{m A T}$, and sAT. Volcano plots revealing the DEGs (FDR $\leq 0.05)$ in obese ( $n=5$ ) versus lean $(n=5)(\mathbf{A})$ eAT, (B) mAT, and $(\mathbf{C})$ sAT and in CAD $(n=6)$ versus NCAD $(n=4)(\mathbf{D})$ eAT, (E) mAT, and (F) sAT. OB, obese; L-, lean.

versus NCAD conditions. Heatmaps representing the individual expression of these genes are given in Supplemental Figures 5, A-C, and these genes' detailed descriptions are given in Supplemental Table 5. Metascape analysis of the upregulated genes pointed toward an activation of inflammation under CAD in mAT with representation of pathways such as neutrophil degradation $(P<0.0001)$, cytokine signaling in immune system $(P<0.001)$, and defense response to bacterium $(P<0.001)$ (Supplemental Figure $5 \mathrm{D})$. Interestingly, CAD was associated with consistent downregulation of $D D X 39 B$ across all fat depots (Figure 3, D-F, and Supplemental Figure 5, A-C). HLAC also exhibited a similar pattern of expression across CAD eAT $\left(\log _{2} \mathrm{FC}=-7.53 ; \mathrm{FDR}=0.053\right), \mathrm{mAT}\left(\log _{2} \mathrm{FC}=-4.97 ; \mathrm{FDR}=0.002\right)$ and sAT $\left(\log _{2}\right.$ FC $=-6.78 ;$ FDR $\left.=0.07\right)($ Supplemental Figure $5, \mathrm{~A}-\mathrm{C}$, and Supplemental Table 5). To understand their relevance to CAD, we analyzed these genes using the STRING platform, which can identify protein-protein interactions based on published reports, experimental studies, and curated databases. A maximum stringent interaction score of 0.9 revealed an interaction network of DDX39B that included proteins such as MAGOH, RBM8A, ALYREF, MAGOHB, and THOC1, all of which functionally associated with KEGG pathways such as spliceosome (ID: 03040; FDR =9.58e-18), RNA transport (ID: 03013; FDR = 2.75e-17), and mRNA surveillance pathway (ID: 03015; FDR = 1.72e-09) (Supplemental Figure 5E and Supplemental Table 8). Similarly, HLAC protein-protein interaction 
network included members such as KIR2DL3, KIR2DL1, B2M, and TAPBP that are involved in KEGG pathways such as antigen processing and presentation (ID: 04612; FDR $=1.43 \mathrm{e}-22$ ), graft-versushost disease (ID: 05332; FDR = 8.44e-19), and cellular senescence (ID: 04218; FDR = 3.46e-12) (Supplemental Figure 5F and Supplemental Table 9).

UCP1-related major transcriptomic shift was specific to eAT. RNA sequencing confirmed our criteria for sample selection because UCP1 expression was significantly different in eAT when patients were segregated into high- and low-UCP1 expression groups ( $n=5$ each, and $P \leq 0.05$ ) (Supplemental Table $1 \mathrm{E}$ ). No other differences in the anthropometric or biochemical features were observed in the high- versus low-UCPI groups (Supplemental Figure 1E).

Using threshold criteria of FC greater than or equal to 2 or less than or equal to -2 and FDR less than or equal to 0.05 revealed 203, 8, and 0 DEGs in the high- versus low-UCP1 groups for eAT (Figure 4A), mAT (Figure 4B), and sAT (Figure 4C), respectively. Clearly, most of the UCP1-related transcriptomic shift occurred in eAT alone (Figure 4, A-C, and Supplemental Figure 6, A and B). Although sAT exhibited no DEGs, mAT exhibited an upregulation in DUXAP8, a HOX family pseudogene, and a downregulation in SNORA73B, SNORA74B, SUSD4, CDH3, CCL19, SLCO5A1, and SCARNA10, genes representing small nucleolar RNA and immune factors in the mAT of patients exhibiting high $U C P 1$ expression in their respective eAT depots (Supplemental Figure 6B and Supplemental Table 6).

In the case of eAT, 27 DEGs were upregulated, and 176 were downregulated in patients with high expression of UCP1 relative to the patients with low expression of UCP1 (Supplemental Figure 6A and Supplemental Table 6). Interestingly, IPA's CPA tool revealed that high expression of UCP1 was associated with a downregulation of multiple immune-related pathways in eAT (Figure 4D). Indeed, a number of these pathways, such as T cell receptor signaling, iCOS-iCOSL signaling in T helper cells, Th2 pathway, $\mathrm{T}$ helper cell differentiation, calcium-induced $\mathrm{T}$ lymphocyte apoptosis, and $\mathrm{B}$ cell receptor signaling, were overrepresented in eAT relative to $\mathrm{mAT}$ and sAT (Figure 2A), indicating that although a generalized activation of the immune system is characteristic of eAT, UCP1 expression is associated with a specific downregulation of these pathways within eAT.

High expression of UCP1 in eAT was also associated with a downregulation of TNFA, identified by the Upstream Regulator Analysis tool in IPA (Supplemental Figure 6D). All the genes TNFA regulates were downregulated in eAT with high expression of $U C P 1$, which exhibited a similar trend upon qPCR validation (Supplemental Figure 6, E-H). Similarly, when IPA's Diseases and Functions tool was used, a clear inhibition in multiple immune-related functions, in particular $\mathrm{T}$ cell-related processes, and downregulation of the production of reactive oxygen species was observed in high versus low UCP1 expression in eAT (Figure 5). Indeed, most genes associated with these functions (except for $H B A 1 / 2$ and NOTCH4) were downregulated in eAT with high expression of UCP1. Besides UCP1, $A C T G 2$ and NOTCH4 were among the top upregulated genes, and GTSF1 and the TRAJ family of genes (i.e., TRAJ12, TRJA29, TRAJ38, and TRAJ17) were among the top downregulated genes in high- versus low-UCP1 groups in eAT (Supplemental Table 6). Expression of a variety of these genes was further verified using a qPCR approach (Supplemental Figures 7-9), where the majority exhibited an expression pattern consistent with downregulation of immune- and redox-related processes in eAT samples with high expression of UCP1.

To address whether these changes were related to the UCP1 expression in eAT per se, we performed Pearson's correlation analysis between UCP1 and the rest of the genes identified by RNA sequencing for eAT, which short-listed 2,845 genes exhibiting $P$ value less than or equal to 0.05 and a positive correlation with $U C P 1(r \geq 0.5)$ and 2,530 genes exhibiting $P$ value less than or equal to 0.05 and a negative correlation with $U C P 1(r \leq-0.5)$. Heatmaps representing the top 10 genes exhibiting significant positive and negative correlations with $U C P 1$ in eAT are shown in Supplemental Figure 6C, and their detailed description is given in Supplemental Table 7. IPA's CPA tool further revealed that genes exhibiting a positive correlation with $U C P 1$ associated with thermogenesis-related pathways, such as mitochondrial dysfunction $(P<0.0001)$, oxidative phosphorylation $(P<0.0001)$, sirtuin signaling $(P<$ $0.0001)$, and TCA cycle II $(P<0.0001)$, whereas negatively correlated genes predominantly associated with immune-related responses, especially T cell-related pathways $(P<0.0001)$ (Figure $4 \mathrm{E})$, verifying our observations that high expression of $U C P 1$ in eAT particularly associates with downregulation of $\mathrm{T}$ cell-related processes in eAT. 


\section{Discussion}

Having established that UCP1 expression is a variable yet consistent feature of human eAT previously (15), we sought to identify the UCP1-associated transcriptomic signatures of human eAT in the current study by specifically selecting patients with high and low expression of UCP1 in eAT from our previous cohort. Paired mAT and sAT samples were also included for each patient to look at depot-specific differences. In addition, our patient selection allowed us to characterize the obesity- and CAD-associated transcriptomic shifts in each of the adipose tissues under study. Consistent with our previous observations where adrenergic stimulation of eAT was associated with specific downregulation of secreted proteins related to immune and oxidative stress pathways in the in vitro settings (15), here we show that high UCP1 expression in eAT relates with downregulation of immune-related pathways, in particular T cell-related processes, as well as reactive oxygen species generation at the whole-transcriptome level.

At the depot level, both eAT and mAT as visceral fat depots shared a transcriptomic profile consistent with an overrepresentation of immune-related processes relative to sAT, in line with a previous study (19). However, quite interestingly, eAT exhibited another layer of depot specificity with an overrepresentation of immune, in particular, $\mathrm{T}$ cell-associated processes from activation and differentiation to apoptosis, relative to both $\mathrm{mAT}$ and sAT. Indeed, an upregulation in the expression of 55 genes associated with inflammation in human eAT relative to omental adipose tissue has been reported before (20). However, a previous study directly comparing eAT, mAT, and sAT using whole-genome microarrays $(n=6)$ did not observe much distinction in eAT and $\mathrm{mAT}$, finding both of them to overexpress inflammatory signals relative to sAT (19). Indeed, a variable transcriptomic profile for eAT based on its anatomic location around the heart has been reported before, where an inflammatory profile was specifically associated with periventricular eAT as opposed to periatrial and peri-coronary artery eAT (21). Thus, distinction in adipose tissue sample collection site, sample size, as well as the sensitivity of RNA sequencing over microarray platforms might have contributed to the observed differences.

An overrepresentation of immune-related gene expression in eAT relative to sAT has been reported before (21-26), and most of these studies attributed this inflammatory profile to the presence of CAD. A recent study, however, revealed that an enrichment of inflammatory signals in eAT is a generic phenomenon that is evident irrespective of CAD status (25). We showed that an increased inflammatory profile of eAT was not associated with either CAD or obesity status in our patients. Low-grade inflammation has been associated with proper adipose tissue differentiation and function in animal studies (27). The observed overrepresentation of immune factors in eAT, however, likely points to a unique depot-specific phenotype that allows eAT to serve its physiological functions. Specific immune factors and adipocytokines within eAT might have a specific role to play in the regulation of cardiac function. Contrarily, its immune phenotype might reflect the predisposition of eAT to be affected by the pathophysiological changes in the cardiovascular apparatus owing to its anatomic proximity (4). Indeed, CAD presence has been shown to augment the inflammatory profile of eAT $(24,25)$.

Besides immune phenotype, eAT also segregated from mAT and sAT based on the specific upregulation in TCF21 and TBX20, transcription factors primarily known to be expressed in cardiac progenitor cells and to be involved in heart development but that have recently been ascribed additional roles in cardiac pathophysiology $(28,29)$. In fact, TCF21 was recently identified as a gene associated with CAD in genome-wide association studies (28). Its overexpression in eAT has also been reported previously (30). In addition, eAT specifically exhibited downregulation in the HOX family of genes, which are associated with regional variation of adipose tissue but are generally expressed in both visceral and subcutaneous adipose tissues (31). Indeed, HOX family gene expression was observed in both mAT and sAT in the current study. It is important to note that $\mathrm{HOXC} 9$ expression is particularly associated with the white phenotype of a fat depot. We previously reported downregulation in $H O X C 9$ expression in eAT relative to both mAT and sAT (15), a pattern that was repeated here.

Obesity is often associated with an increase in inflammation, especially M1-polarized macrophage-mediated proinflammatory markers, extracellular matrix remodeling factors, and downregulation in fatty acid and mitochondrial energy metabolism (32), sometimes to a similar extent in SAT and visceral fat depots (33). Indeed, genomic or transcriptomic studies where gene expression changes can be specifically attributed to obesity in eAT and mAT are currently lacking. We observed a unique pattern of obesity-related transcriptomic shift that was exclusive to sAT. However, it is important to acknowledge that our observations in sAT cannot be specifically attributed to obesity either. Although no changes in the observed biochemical 
A

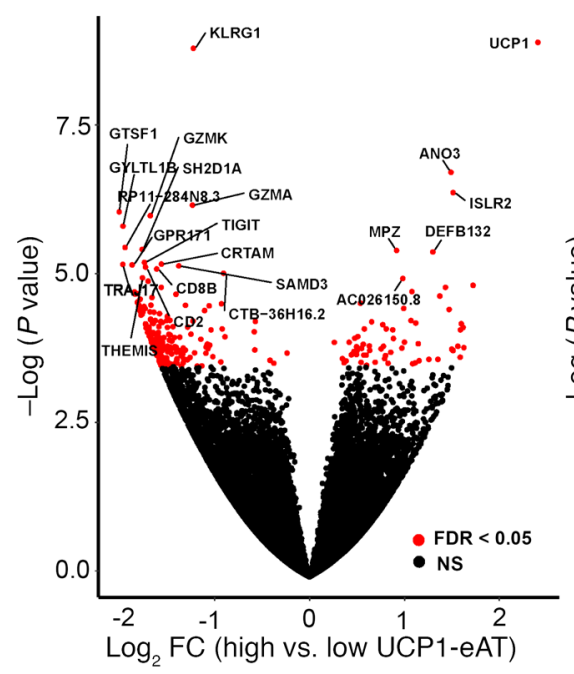

B

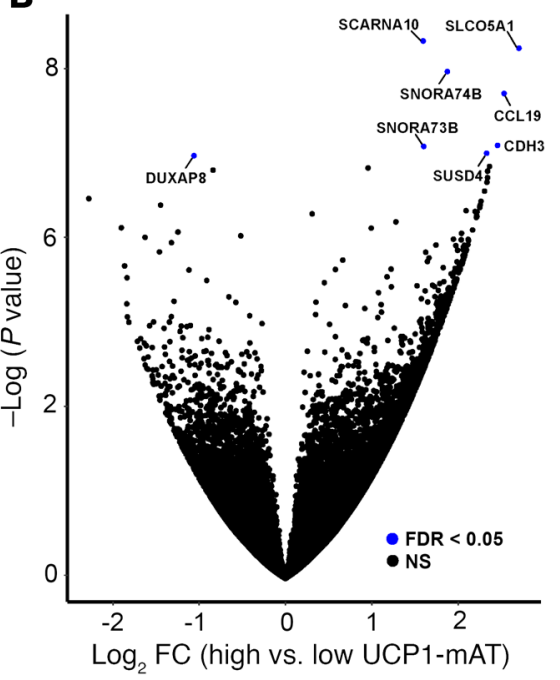

C

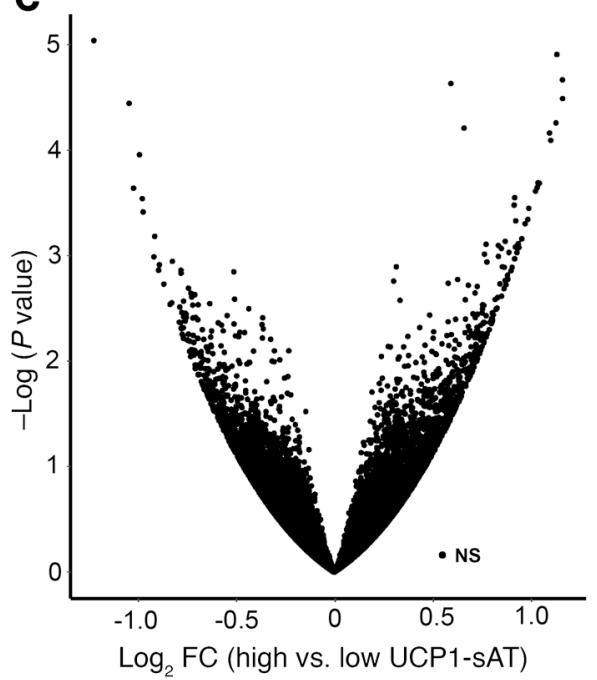

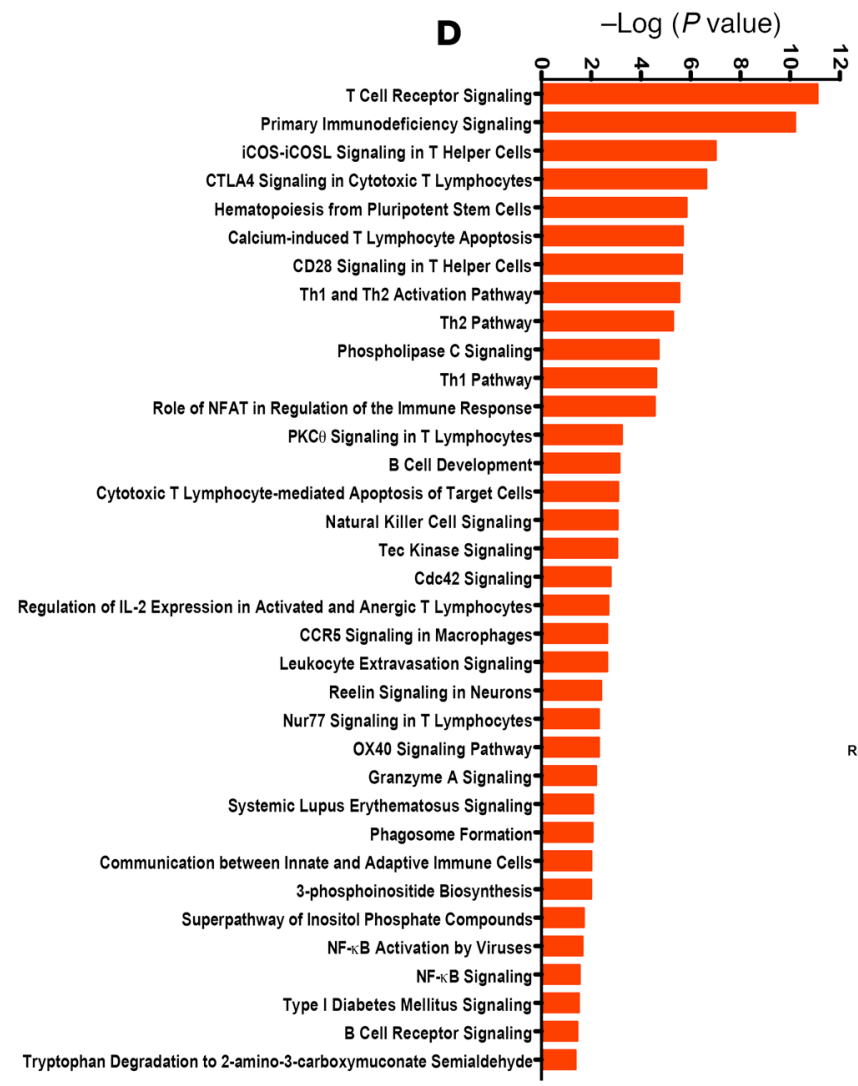

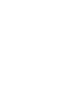

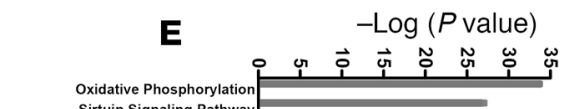

Sirtuin Signaling Pathway CXCR4 Signaling Junction Signalin Thrombin Signaling

CDK5 Signaling
Breast Cancer Regulation by Stathmin 1 Breast Cancer Regulation by Stathmin

Cardiac Hypertrophy Signaling TCA Cycle II (Eukaryotic) Sertoli Cell-Sertoli Cell Junction Signaling Itegrin Signaling
ILK Signaling Histamine Degradatio edullin signaling pathwa Ethanol Degradation IV Junction Signaling Relaxin Signaling I Guidance Signalin Tight Junction Signalin Alpha-Adrenergic Signalin Ephrin Receptor Signaling Ephrin B Signaling T Cell Receptor Signali PS-icosL Signaling in T Helper Cell Primary Immunodeficiency Signalin
CD28 Signaling in T Helper Cells BRCA1 in DNA I PI3K Signaling in B Lymphocytes -induced T Lymphocyte Apoptosis CTLA4 Signaling in Cytotoxic T Lymphocytes PKC_Signaling in T Lymphocytes Regulation of IL-2 Expression in Activated and Anergic T Lymphocytes B Cell Receptor Signaling DNA Double-Strand Break Repair by Homologous Recombination Systemic Lupus Erythematosus Signaling
Glutamine Degradation I Th1 Pathway hospholipase C Signaling h1 1 and Th2 Activation Pathway Sumoylation Pathway
Sula Fc Epsilon RI Signaling ATM Signaling

Figure 4. UCP1-associated transcriptomic differences in eAT, $\mathbf{m A T}$, and sAT. Volcano plots revealing the DEGs (FC $\geq 2$ or $\leq-2$; FDR $\leq 0.05)$ in (A) eAT, (B) mAT, and (C) sAT when patients were segregated based on high versus low UCP1 expression in eAT. IPA's canonical pathways exhibiting Benjamini-Hochberg-corrected $P$ less than or equal to 0.05 , identified by (D) differentially downregulated genes in high versus low UCP1 expression in eAT, and by (E) positively ( $r \geq 0.50$, and $P \leq 0.05)$ UCP1-correlated and negatively ( $r \leq-0.50$, and $P \leq 0.05)$ UCP1-correlated with UCP1 in eAT alone. A Pearson's correlation analysis was applied to UCP1 and the rest of the genes in the eAT depot $(n=10)$.

parameters or age were observed between lean versus obese patients (Supplemental Table 1C), other factors associated with their clinical or drug status might have contributed to our observations. Despite these limitations, obesity-associated observations in SAT were consistent with previous reports (32).

Similar to obesity status, CAD status did not result in specific transcriptomic shift in eAT, while multiple studies have revealed CAD-associated changes in human eAT $(25,26)$, even with the microarray studies $(22,34)$. Increased expression of $11 B H S D, G C R$, and $C D 68$ has also been reported in mAT of 


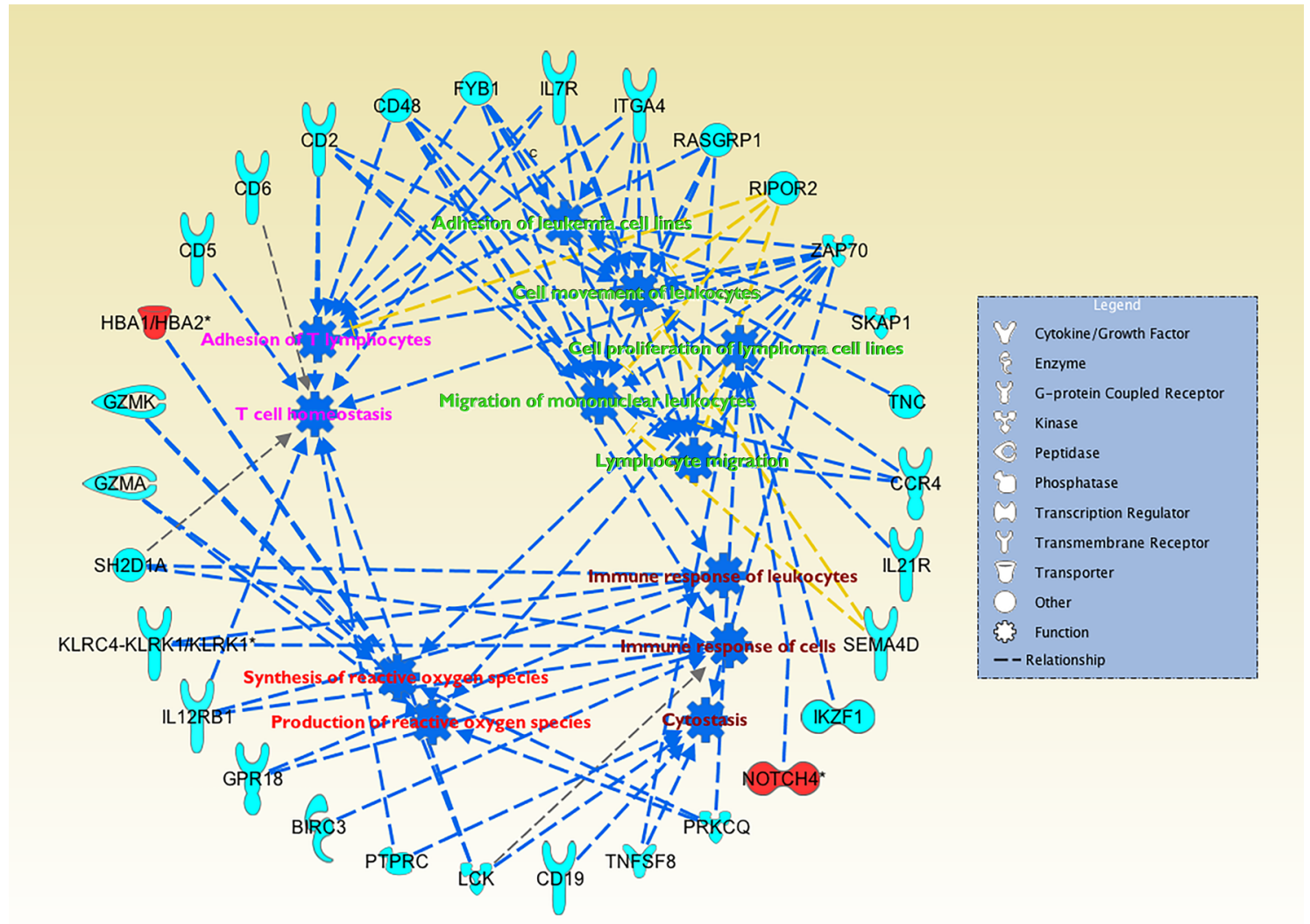

() 2000-2018 QIAGEN. All rights reserved.

Figure 5. Proposed functional relevance of UCP1 in eAT. Network revealing functional relevance of downregulated genes in high versus low UCP1 expression in eAT.

patients with $\mathrm{CAD}$ compared with $\mathrm{NCAD}$, pointing toward a pathogenic immune profile of mAT during CAD (35). We also observed an upregulation in immune-related pathways in CAD versus NCAD mAT. In addition, we observed consistent downregulation in $D D X 39 B$ and $H L A C$ genes across all fat depots. A genome-wide meta-analysis study identified an MHC locus to be associated with CAD; however, it could not be attributed to the $H L A C$ allele specifically (36). Considering that MHC genes regulate inflammation and $\mathrm{T}$ cell responses that are involved in the initiation and propagation of atherosclerosis, future studies focusing on the role of these alleles in CAD are warranted. In addition, we identified a potentially novel gene, $D D X 39 B$, to be differentially downregulated across all the fat depots in CAD. Although little is known about $D D X 39 B$, network analysis revealed a role for $D D X 39 B$ and other genes in its network were associated with mRNA transport and surveillance pathways. Its role in adipose tissue physiology and the etiology of CAD remains to be discerned.

Exploration of UCP1-associated gene signatures in eAT, mAT, and sAT revealed most transcriptomic changes to be located in eAT alone. We $(8,15)$, and others $(7,21,30)$, have reported specific upregulation in UCP1 expression in human eAT relative to both $\mathrm{mAT}$ and sAT. In addition, we recently ascribed a beige phenotype and functional relevance to UCP1 presence within human eAT (15). Thus, it is not surprising to see specific transcriptomic shift located in eAT alone, especially when our patients were selected based on their UCP1 expression within eAT. Quite interestingly, however, we also observed specific downregulation in genes associated with multiple immune-related pathways, especially those related to $\mathrm{T}$ cell responses and adaptive immunity, in patients with high UCP1 expression versus low UCP1 expression in eAT. Similar observations were repeated when positively and negatively $U C P 1$-correlated genes were examined, where thermogenic processes were evident for positively UCP1-correlated genes, and immune responses were abundantly represented by negatively $U C P 1$-correlated genes. A role for adaptive immunity including macrophages (37, 38), type 2 immunity $(39,40)$, and Tregs (41) in beige adipogenesis has been demonstrated previously, where an antiinflammatory immune microenvironment in adipose tissue was associated with upregulation as well as stimulation of beige and thermogenic phenotypes in SAT. Overall, experimental studies have revealed a 
negative association between $U C P 1$ activation and adipose tissue inflammation levels. We provide what may be the first evidence of this relationship in human adipose tissue at the whole-transcriptome level. Indeed, a causal role for UCP1 in the regulation of inflammation in human eAT remains to be established.

Similarly, associations between $U C P 1$ induction and activity with mitochondrial reactive oxygen species production (42) and glutathione levels (43) have been reported in rodent brown and white adipose depots previously. Browning of white adipose tissue has also been suggested to occur as an adaptive mechanism to alleviate redox pressure (44). Increased expression of oxidative stress-related proteins has also been reported in eAT relative to SAT in patients undergoing cardiac surgeries (45), an increase in reactive oxygen species being further associated with reduced expression of UCP1 and related thermogenic markers in the eAT of patients with CAD (46). We also recently observed a clear downregulation in secreted proteins associated with the GO term cell-redox homeostasis in human eAT upon adrenergic stimulation (15). Immune-related processes were the only other GO terms identified in association with adrenergically stimulated, eAT-secreted proteins in our previous study (15). Indeed, our cumulative observations from this and the previous study (15) indicate tight-knit associations among thermogenic properties, regulation of redox status, and immune environment of adipose tissue, thereby supporting a plausible functional role for UCP1 in the regulation of tissue homeostasis in human eAT.

In conclusion, we have observed that eAT exhibits specific upregulation in inflammatory processes as an adipose depot, which are susceptible to accentuation during CAD and other metabolic disturbances. $U C P 1$, likely through its thermogenic function, is able to mitigate these imbalances in the immune and redox environment of human eAT. Indeed, UCP1 can be targeted to maintain eAT homeostasis to manage $\mathrm{CAD}$; however, much work is first needed to establish a causal role for UCP1 in modulating specific immune- and redox-related pathways.

Limitations and strengths of the study. A primary limitation of the study is that it was conducted using samples that were derived from older patients with multiple metabolic abnormalities who came in for various cardiac surgeries. Because the amount of eAT around the human heart is highly variable and dissecting too much eAT is not ideal, our biopsy size was at times quite small, affecting RNA quality and availability for each sample, thereby restricting our sample selection for RNA sequencing from our initial cohort $(n=53)$. Indeed, we also acknowledge that we had a rather small sample size especially when samples were subgrouped per their obesity, CAD, and UCP1 status $(n=4-6)$, which may have contributed to the lack of CAD-associated transcriptomic shifts that were previously observed for human eAT. However, it is important to highlight that our high- versus low-UCP1 samples came from a well-characterized larger cohort $(n=53)(15)$, where adipose depots were collected in a pairwise manner for each patient, thereby allowing us to comment upon direct depot-specific transcriptomic shifts in general as well as upon subgrouping. Finally, our overall observations are in line with reported literature and our recent work that used various in vitro approaches to assess the role of UCP1 in human eAT, thereby providing substantial support to our conclusions.

\section{Methods}

Study population and biopsy collection. Patients undergoing various heart surgeries (e.g., coronary artery bypass grafting and valve replacement) at the IUCPQ participated in the study. Adipose tissue biopsies from 3 compartments, namely epicardial, mediastinal, and subcutaneous, were taken from the chest of each individual. eAT corresponds to the adipose depot in direct contact with the heart located between the myocardium and the visceral pericardium and was sampled from the right ventricle epicardium. mAT was sampled from the fat within the mediastinum outside the pericardial sac. sAT was sampled from the anterior thorax after sternotomy. Detailed patient features and inclusion/exclusion criteria are described elsewhere (15).

UCP1 mRNA expression levels were assessed using quantitative PCR in 53 subjects. From this cohort 10 patients exhibiting high or low expression of $U C P 1$ ( $n=5$ each) were chosen based on sample availability and RNA quality. Age, sex, anthropometric data, as well as clinical and biochemical details of these patients are given in Supplemental Table 1, A and B. Individuals with a BMI less than or equal to $25 \mathrm{~kg} / \mathrm{m}^{2}$ were considered lean, whereas those with a BMI greater than or equal to $30 \mathrm{~kg} / \mathrm{m}^{2}$ were identified as obese. Individuals with coronary artery bypass grafting and a history of myocardial ischemia or coronary artery stenosis greater than $50 \%$ on coronary angiography were identified as CAD patients, whereas those operated on for valve replacement and coronary artery stenosis less than $50 \%$ on coronary angiography were identified as NCAD patients. 
$R N A$ extraction and quality assessment. Total RNA was isolated from adipose tissue biopsies using the RNeasy Lipid Tissue Mini Kit (QIAGEN) according to the manufacturer's instructions. Purity of total RNA was determined as 260/280 $\mathrm{nm}$ absorbance ratio with expected values between 1.8 and 2.0 using Biodrop Duo (BioDrop). In addition, RNA integrity of samples was assessed using the Agilent 2100 Bioanalyzer (Agilent Technologies).

Next-generation RNA sequencing. RNA library preparations were carried out on $500 \mathrm{ng}$ of RNA with RNA integrity number greater than 7 using the Illumina TruSeq Stranded Total RNA Sample Preparation Kit, according to manufacturer's protocol. Final libraries were analyzed on a Bioanalyzer and sequenced on the Illumina HiSeq2500 (pair-ended 125-bp sequences). Raw reads were trimmed for quality (phred33 $\geq 30$ ) and length $(n \geq 32)$, and Illumina adapters were clipped off using Trimmomatic v. 0.35 (PubMed identifier [PMID]: 24695404). Filtered reads were aligned to the GRCh37 human reference using TopHat v. 2.0.14 (PMID: 236184080). Raw read counts of genes were obtained using htseq-count v. 0.6.1 (PMID: 25260700). Differential expression analysis was done using DESeq2 (PMID: 25516281). Raw and processed sequencing data were deposited into the MINSEQE-compliant National Center for Biotechnology Information Gene Expression Omnibus database (GEO GSE125856).

Pathway and network analyses. QIAGEN's IPA (QIAGEN Inc., https://www.qiagenbioinformatics. $\mathrm{com} /$ products/ingenuity-pathway-analysis) was used to derive biologically relevant information from the DEGs. Canonical pathways with Benjamini-Hochberg-corrected $P$ values lower than 0.05 were considered significant (47). In addition, Metascape v. 3.0 was used (http://www.metascape.org) to identify GO biological processes (48), and the STRING platform was used to identify protein-protein interactions as needed (49). Volcano plots and heatmaps were prepared using R.

Validation using $q P C R$. Shortlisted genes were assayed using a qPCR approach where expression of each gene was normalized to the geometric mean of the reference genes PPIA, GAPDH, and RPL27 as described previously (15).

Statistics. Data are presented as mean \pm SEM. Statistical tests were performed using GraphPad Prism for comparing patient characteristics and gene expression analysis (described in detail in the legends), and a $P$ value less than or equal to 0.05 was considered significant. DESeq2-derived differential expression was considered significant for genes exhibiting FDR less than or equal to 0.05. Benjamini-Hochberg-adjusted $P$ value less than or equal to 0.05 was considered significant for pathway analysis.

Study approval. The institutional ethics committee of IUCPQ, Chemin Sainte-Foy, Québec, Canada, approved the study. Written informed consent documents were received from participants before their inclusion in the study.

\section{Author contributions}

$\mathrm{KC}$ and DR conceived and designed the study. KC conducted tissue collection, RNA isolation and quality tests, and data analysis, including pathway and network analyses, and wrote the manuscript. PV provided the biopsies during cardiovascular surgeries. JV and EG sorted raw next-generation sequencing data and performed DESeq2 analysis. YB provided access to IPA and related technical help. KC, DR, PM, YB, AT, and EG held discussions and provided critical reviews of the manuscript.

\section{Acknowledgments}

KC was supported by a Canadian Institute of Health Research Banting postdoctoral fellowship and Fonds de Recherche du Québec-Santé (FRQS) postdoctoral fellowship awards. PM holds an FRQS Research Chair on the Pathobiology of Calcific Aortic Valve Disease.

Address correspondence to: Denis Richard, IUCPQ-UL, 2725 chemin Sainte-Foy, Québec, Québec, G1V 4G5, Canada. Phone: 418.656.8711, ext. 3982; Email: Denis.Richard@criucpq.ulaval.ca.

1. Iacobellis G, Corradi D, Sharma AM. Epicardial adipose tissue: anatomic, biomolecular and clinical relationships with the heart. Nat Clin Pract Cardiovasc Med. 2005;2(10):536-543.

2. Chechi K, Richard D. Thermogenic potential and physiological relevance of human epicardial adipose tissue. Int J Obes Suppl. 2015;5(suppl 1):S28-S34.

3. McAninch EA, et al. Epicardial adipose tissue has a unique transcriptome modified in severe coronary artery disease. Obesity (Silver Spring). 2015;23(6):1267-1278.

4. Iacobellis G, Bianco AC. Epicardial adipose tissue: emerging physiological, pathophysiological and clinical features. Trends Endocrinol Metab. 2011;22(11):450-457. 
5. Aldiss P, Davies G, Woods R, Budge H, Sacks HS, Symonds ME. 'Browning' the cardiac and peri-vascular adipose tissues to modulate cardiovascular risk. Int J Cardiol. 2017;228:265-274.

6. Iacobellis G. Local and systemic effects of the multifaceted epicardial adipose tissue depot. Nat Rev Endocrinol. 2015;11(6):363-371

7. Sacks HS, et al. Uncoupling protein-1 and related messenger ribonucleic acids in human epicardial and other adipose tissues: epicardial fat functioning as brown fat. J Clin Endocrinol Metab. 2009;94(9):3611-3615.

8. Chechi K, Blanchard PG, Mathieu P, Deshaies Y, Richard D. Brown fat like gene expression in the epicardial fat depot correlates with circulating HDL-cholesterol and triglycerides in patients with coronary artery disease. Int J Cardiol. 2013;167(5):2264-2270.

9. Cannon B, Nedergaard J. Brown adipose tissue: function and physiological significance. Physiol Rev. 2004;84(1):277-359.

10. Bartelt A, et al. Brown adipose tissue activity controls triglyceride clearance. Nat Med. 2011;17(2):200-205.

11. Bartelt A, et al. Thermogenic adipocytes promote HDL turnover and reverse cholesterol transport. Nat Commun. 2017;8:15010.

12. Cypess AM, et al. Anatomical localization, gene expression profiling and functional characterization of adult human neck brown fat. Nat Med. 2013;19(5):635-639.

13. Lidell ME, et al. Evidence for two types of brown adipose tissue in humans. Nat Med. 2013;19(5):631-634.

14. Jespersen NZ, et al. A classical brown adipose tissue mRNA signature partly overlaps with brite in the supraclavicular region of adult humans. Cell Metab. 2013;17(5):798-805.

15. Chechi K, et al. Functional characterization of the Ucp1-associated oxidative phenotype of human epicardial adipose tissue. Sci Rep. 2017;7(1):15566

16. Gaborit B, et al. Human epicardial adipose tissue has a specific transcriptomic signature depending on its anatomical peri-atrial, peri-ventricular, or peri-coronary location. Cardiovasc Res. 2015;108(1):62-73.

17. Fain JN, et al. Identification of omentin mRNA in human epicardial adipose tissue: comparison to omentin in subcutaneous, internal mammary artery periadventitial and visceral abdominal depots. Int J Obes (Lond). 2008;32(5):810-815.

18. Stewart A, Guan H, Yang K. BMP-3 promotes mesenchymal stem cell proliferation through the TGF-beta/activin signaling pathway. J Cell Physiol. 2010;223(3):658-666.

19. Guauque-Olarte S, et al. The transcriptome of human epicardial, mediastinal and subcutaneous adipose tissues in men with coronary artery disease. PLoS One. 2011;6(5):e19908.

20. Imoto-Tsubakimoto $\mathrm{H}$, et al. Serglycin is a novel adipocytokine highly expressed in epicardial adipose tissue. Biochem Biophys Res Commun. 2013;432(1):105-110.

21. Gaborit B, et al. Human epicardial adipose tissue has a specific transcriptomic signature depending on its anatomical peri-atrial, peri-ventricular, or peri-coronary location. Cardiovasc Res. 2015;108(1):62-73.

22. Mazurek T, et al. Human epicardial adipose tissue is a source of inflammatory mediators. Circulation. 2003;108(20):2460-2466.

23. Hirata Y, et al. Coronary atherosclerosis is associated with macrophage polarization in epicardial adipose tissue. $J$ Am Coll Cardiol. 2011;58(3):248-255.

24. Shimabukuro M, et al. Epicardial adipose tissue volume and adipocytokine imbalance are strongly linked to human coronary atherosclerosis. Arterioscler Thromb Vasc Biol. 2013;33(5):1077-1084.

25. Vacca M, et al. Integrative miRNA and whole-genome analyses of epicardial adipose tissue in patients with coronary atherosclerosis. Cardiovasc Res. 2016;109(2):228-239.

26. Sacks HS, et al. Depot-specific overexpression of proinflammatory, redox, endothelial cell, and angiogenic genes in epicardial fat adjacent to severe stable coronary atherosclerosis. Metab Syndr Relat Disord. 2011;9(6):433-439.

27. Wernstedt Asterholm I, et al. Adipocyte inflammation is essential for healthy adipose tissue expansion and remodeling. Cell Metab. 2014;20(1):103-118.

28. Nurnberg ST, et al. Coronary artery disease associated transcription factor TCF21 regulates smooth muscle precursor cells that contribute to the fibrous cap. PLoS Genet. 2015;11(5):e1005155.

29. Kirk EP, et al. Mutations in cardiac T-box factor gene TBX20 are associated with diverse cardiac pathologies, including defects of septation and valvulogenesis and cardiomyopathy. Am J Hum Genet. 2007;81(2):280-291.

30. Sacks HS, et al. Adult epicardial fat exhibits beige features. J Clin Endocrinol Metab. 2013;98(9):E1448-E1455.

31. Passaro A, et al. Gene expression regional differences in human subcutaneous adipose tissue. BMC Genomics. 2017;18(1):202.

32. Fuster JJ, Ouchi N, Gokce N, Walsh K. Obesity-induced changes in adipose tissue microenvironment and their impact on cardiovascular disease. Circ Res. 2016;118(11):1786-1807.

33. Klimcáková E, et al. Worsening of obesity and metabolic status yields similar molecular adaptations in human subcutaneous and visceral adipose tissue: decreased metabolism and increased immune response. J Clin Endocrinol Metab. 2011;96(1):E73-E82.

34. Dutour A, et al. Secretory type II phospholipase A2 is produced and secreted by epicardial adipose tissue and overexpressed in patients with coronary artery disease. J Clin Endocrinol Metab. 2010;95(2):963-967.

35. Atalar F, et al. Mediastinal adipose tissue expresses a pathogenic profile of $11 \beta$-hydroxysteroid dehydrogenase Type 1 , glucocorticoid receptor, and CD68 in patients with coronary artery disease. Cardiovasc Pathol. 2013;22(3):183-188.

36. Davies RW, et al. A genome-wide association study for coronary artery disease identifies a novel susceptibility locus in the major histocompatibility complex. Circ Cardiovasc Genet. 2012;5(2):217-225.

37. Lee MW, et al. Activated type 2 innate lymphoid cells regulate beige fat biogenesis. Cell. 2015;160(1-2):74-87.

38. Nguyen KD, et al. Alternatively activated macrophages produce catecholamines to sustain adaptive thermogenesis. Nature. 2011;480(7375):104-108.

39. Qiu Y, et al. Eosinophils and type 2 cytokine signaling in macrophages orchestrate development of functional beige fat Cell. 2014;157(6):1292-1308

40. Rao RR, et al. Meteorin-like is a hormone that regulates immune-adipose interactions to increase beige fat thermogenesis. Cell. 2014;157(6):1279-1291.

41. Brestoff JR, et al. Group 2 innate lymphoid cells promote beiging of white adipose tissue and limit obesity. Nature. 2015;519(7542):242-246.

42. Chouchani ET, et al. Mitochondrial ROS regulate thermogenic energy expenditure and sulfenylation of UCP1. Nature. 2016;532(7597):112-116. 
43. Lettieri Barbato D, Tatulli G, Maria Cannata S, Bernardini S, Aquilano K, Ciriolo MR. Glutathione decrement drives thermogenic program in adipose cells. Sci Rep. 2015;5:13091.

44. Carrière A, et al. Browning of white adipose cells by intermediate metabolites: an adaptive mechanism to alleviate redox pressure. Diabetes. 2014;63(10):3253-3265

45. Salgado-Somoza A, Teijeira-Fernández E, Fernández AL, González-Juanatey JR, Eiras S. Proteomic analysis of epicardial and subcutaneous adipose tissue reveals differences in proteins involved in oxidative stress. Am J Physiol Heart Circ Physiol. 2010;299(1):H202-H209.

46. Dozio E, et al. Increased reactive oxygen species production in epicardial adipose tissues from coronary artery disease patients is associated with brown-to-white adipocyte trans-differentiation. Int J Cardiol. 2014;174(2):413-414.

47. Krämer A, Green J, Pollard J Jr, Tugendreich S. Causal analysis approaches in Ingenuity Pathway Analysis. Bioinformatics. 2014;30(4):523-530

48. Tripathi S, et al. Meta- and orthogonal integration of influenza "OMICs" data defines a role for UBR4 in virus budding. Cell Host Microbe. 2015;18(6):723-735.

49. Szklarczyk D, et al. The STRING database in 2017: quality-controlled protein-protein association networks, made broadly accessible. Nucleic Acids Res. 2017;45(D1):D362-D368. 